\title{
Expression of Glypican-3 is Highly Associated with Pediatric Hepatoblastoma: a Systemic Analysis
}

\author{
Xiao-Li Xiong ${ }^{1,2 \&}$, Huan Qin ${ }^{2 \&}$, Su-Qi Yan ${ }^{2}$, Li-Shan Zhou ${ }^{2}$, Peng Chen ${ }^{2}$, Dong- \\ Chi Zhao'*
}

\begin{abstract}
Objective: Glypican-3 (GPC3) is reported to be an oncofetal protein that is a useful diagnostic immunomarker for hepatoblastoma. However, the results are not inclusive. This study systemically investigated the association between expression of GPC3 and pediatric hepatoblastoma. Methods: Clinical studies evaluating the association were identified using a predefined search strategy. GPC 3 immunohistochemistry was applied in the pathological diagnosis of hepatoblastoma using the monoclonal antibodies with formalin-fixed and paraffin-embedded specimens. Positive predictive rates for the association between expression of GPC3 and pediatric hepatoblastoma were calculated. Results: Specimens from four clinical studies which including 134 patients with pediatric hepatoblastoma tested by GPC3 immunohistochemistry were considered eligible for inclusion. Systemic analysis showed that, in all patients, pooled positive predictive rate of the association between expression of GPC3 and pediatric hepatoblastoma was $95.5 \%(128 / 134)$. Conclusion: This systemic analysis suggests that the expression of glypican-3 is highly associated with the diagnosis of pediatric hepatoblastoma.
\end{abstract}

Keywords: Glypican-3 - hepatoblastoma

Asian Pac J Cancer Prev, 16 (3), 1029-1031

\section{Introduction}

Hepatoblastoma is one of the most common primary cancers in children and accounts for two-thirds of all malignant liver neoplasms in children (Ismail et al., 2012). The risk factor of pediatric hepatoblastoma $(\mathrm{PH})$ is mostly unclear.

The diagnosis of liver nodular disease is always elusive and sometimes difficult for pathologists. Although reticulin staining and (more recently) CD34-immunohistochemistry have been recognized as helpful diagnostic markers in differential diagnoses among hepatic lesions (hepatocellular carcinoma, dysplastic nodules, and other benign hepatocellular lesions), their clinical effectiveness is still somewhat not clear (Park et al., 1998; Kong et al., 2000). Another difficult challenge in the diagnosis of hepatocellular carcinoma is the differentiation from primary hepatic cholangiocellular carcinoma and liver metastases. Differentiation markers for immunohistochemistry have included hepatocyte antigen (Hep), alpha-fetoprotein (AFP), cytokeratin protein profiling, and the presence of intercellular canaliculi demonstrated by the monoclonal antibodies carcinoembryonic antigen Gold 5 and B-lymphocyte marker CD10. As the variability of these markers might suggest, however, their specificity and sensitivity are somewhat limited for the diagnosis of hepatocellular carcinoma (Chu et al., 2002). Glypican 3 (GPC3) is one of the cell surface heparan sulfate proteoglycans that bind to the cell membrane via glycosilphosphatidylinositol anchors (Filmus et al., 2001; Abdelgawad et al., 2013; Kim et al., 2013). Heparan sulfate proteoglycans are known to interact with growth factors through heparan sulfate chains and thereby to serve as coreceptors for heparin-binding growth factors (Grisaru et al., 2001; Paine-Saunders et al., 2000; Zhang et al., 2001). While studies on ovarian cancer cell lines, mesotheliomas, and breast tumors have demonstrated the down regulation of GPC3 (Lin et al., 1999; Murthy et al., 2000; Xiang et al., 2001), other investigations on hepatocellular carcinoma have shown a marked elevation of GPC 3 mRNA over the level observed in corresponding normal tissues (Hsu et al., 1997; Lage et al., 1998; Saikali et al., 2001; Zhu et al., 2001). On this background, we hypothesize that GPC 3 could be associated with the clinical manifestation of pediatric hepatoblastoma.

\section{Materials and Methods}

\section{Search strategy}

We searched PUBMED, by using the following search term: (glypican-3) and (pediatric hepatoblastoma). All 
Xiao-Li Xiong et al

clinical studies evaluating the impact of glypican-3 on the clinical manifestation for pediatric hepatoblastoma published in English prior to December 2014 were identified. If samples of two studies overlap, only the newest one was included. Additional articles were obtained from references within the articles identified by the electronic search. We did not consider meeting abstracts or unpublished reports.

\section{Inclusion and exclusion criteria}

We reviewed abstracts of all citations and retrieved studies. The following criteria were used to include published studies: (1) clinical studies, containing ; (2) The study was performed in accordance with the Helsinki Declaration (1964, amended in 1975 and 1983) of the World Medical Association. Eligibility criteria included histologically or cytologically verified metastatic breast cancer, the presence of at least one bidimensionally measurable lesion, a performance status (WHO) 2, age 18 years. Studies were excluded if one of the following existed: (a) duplicate data; (b) no sufficient data were reported.

\section{Data collection and analysis}

Selection of trials and data extraction: The titles and abstracts of publications identified according to the above search strategy were assessed independently for inclusion by two authors, the full text was selected for further assessment if the abstract suggests relevance. Disagreement was resolved by discussion. Data was extracted by independent authors. The following recorded data were extracted: author, publication data, and country of the first or corresponding author, the number of patients. Outcome measures presented in at least 3 studies were extracted for combined analysis.

\section{Results}

We searched PUBMED, by using the following search term: (glypican-3) and (pediatric hepatoblastoma). There were 285 papers relevant to the search words by the end of November 2014. Via steps of screening the title and reading the abstract, 4 studies were identified (Wang, et al., 2013; Chan et al., 2013; Zynger et al., 2008; Yamauchi et al., 2005). These studies had been carried out in China, Japan, and the USA. The following outcomes were presented in at least all studies and extracted for combined analysis: the relationship between glypican-3 and pediatric hepatoblastoma regarding clinical manifestation, or prognosis. Characteristics of studies included in the analysis are presented as these outcomes: GPC3 was positive in fetal epithelial cells $(54 / 54,100 \%)$, Nonneoplastic hepatocytes adjacent to tumor were negative for GPC3 expression (0/10) of Wang, et al.; GPC3 expression was seen in 9/9 (100\%) hepatoblastomas of Chan et al.; All 65 hepatoblastomas had cytoplasmic immunoreactivity for GPC3 with greater than $90 \%$ of cases showing strong, diffuse positivity of Zynger et al.; and Diffusely positive staining of GPC3 was observed in malignant hepatocytes in hepatoblastomas and in hepatocellular carcinomas $(47 / 56,84 \%)$ of Yamauchi et al. Totally, specimens from four clinical studies which including 134 patients with pediatric hepatoblastoma tested for GPC3 were included. Systemic analysis suggested that pooled positive predictive rate of the association between expression of GPC3 and pediatric hepatoblastoma was 95.5\% (128/134).

\section{Discussion}

Hepatoblastoma is one of the common primary cancers in children. Differentiation markers for hepatoblastoma are suggested to be Hep, AFP, cytokeratin protein profiling, etc. GPC3 is a member of the glypican family of glycosylphosphatidyl inositol-anchored cell-surface heparan sulfate proteoglycans (Filmus et al., 2001). The messenger RNA (mRNA) levels of GPC3 have recently been found to be markedly elevated in a large proportion of hepatocellular carcinomas, and this elevation is apparently independent of AFP (Hsu, et al., 1997; Zhu et al., 2001; Midorikawa et al., 2003). As no GPC3 was detected in the hepatocytes of chronic hepatitis, liver cirrhosis, or normal adult liver, it is suggested that GPC3 could be regarded as an oncofetal protein, at least in the liver. Although GPC3 was originally identified as a suppressor of cell proliferation, 21,22 it also acts as a negative regulator of inhibitory growth factors such as BMP-7 (Piscione et al., 2001; Watanabe et al., 2003). Intracellular distribution of GPC3 might be related to such an altered function. Thus, the GPC 3 expression in malignant hepatocytes appears to indicate not only the reactivation of a fetal phenotype, but possibly some other biologically significant event also in the carcinogenesis of the liver.

One study conducted in China using five tissue microarray paraffin blocks and included 54 patients of hepatoblastoma, in which tumor tissue samples were obtained from 3 surgical biopsies, 33 needle biopsies, 5 stage I resection tumors, and 13 stage II resection tumors after transcatheter arterial chemoembolization. Ten samples of non-neoplastic hepatic tissue adjacent to tumor were used as control (Wang, et al., 2013). In this study, immunohistochemical staining of GPC3 was performed, and among these 54 patients, 22 were fetal subtype, 24 were mixed fetal and embryonal subtype and 8 cases were mixed epithelial and mesenchymal type (Wang, et al., 2013). GPC3 was positive in fetal epithelial cells $(54 / 54,100 \%)$, but negative or weakly positive in embryonic epithelial cells in all cases of hepatoblastoma. Undifferentiated small cells and all mesenchymal components were negative for the expression. Nonneoplastic hepatocytes adjacent to tumor were negative for GPC3 expression (0/10) (Wang, et al., 2013) . In another study conducted in the USA, GPC3 expression in 143 pediatric central nervous system (CNS) tumors and 271 non-CNS tumors was investigated (Chan et al., 2013). In this study, among non-CNS tumors, GPC3 expression was seen in 9/9 (100\%) hepatoblastomas (Chan et al., 2013). In Chicago, GPC3 immunoreactivity was semiquantitatively evaluated in 65 patients with hepatoblastoma (Zynger et al., 2008). In this study, histologic patterns in each tumor were individually assessed for immunoreactivity. All 65 hepatoblastomas had cytoplasmic immunoreactivity for GPC3 with greater than $90 \%$ of cases showing 
strong, diffuse positivity (Zynger et al., 2008). And it was found from this study that no reactivity in benign liver tissue could be tested. Fetal, embryonal, and small cell undifferentiated patterns were diffusely positive in almost all cases, whereas mesenchymal and teratoid patterns were nearly all negative (Zynger et al., 2008). Thus it was concluded from this study that that GPC3 may play a role in the tumorigenesis of hepatoblastoma (Zynger et al., 2008). A Japanese group observed diffusely positive staining of GPC3 in malignant hepatocytes in hepatoblastomas and in hepatocellular carcinomas (Yamauchi et al., 2005). GPC3 expression was independent of the differentiation and size of the hepatocellular carcinoma. On the other hand, there was only weak and focal staining in low-grade (2/8) and high-grade dysplastic nodules (6/8). GPC3 immunoreactivity was detected in only one of 23 metastatic lesions of colorectal carcinoma, and its expression was entirely absent in the liver cell adenoma $(0 / 7)$, carcinoid tumor $(0 / 1)$, and cholangiocellular carcinoma $(0 / 16)$. When compared with immunohistochemistry of hepatocyte antigen and alpha-fetoprotein, GPC3-immunohistochemistry was significantly much more specific and sensitive for hepatocellular carcinomas (Yamauchi et al., 2005). Thus, GPC3 was confirmed to be one of the oncofetal proteins now attracting attention for their promise both as markers of hepatocellular carcinoma in routine histological examination and as targets in monoclonal antibody-based hepatocellular carcinoma therapy (Yamauchi et al., 2005).

Our current systemic analysis revealed that specimens from four clinical studies which including 134 patients with pediatric hepatoblastoma tested for GPC3 were included. Systemic analysis suggested that pooled positive predictive rate of the association between expression of GPC3 and pediatric hepatoblastoma was $95.5 \%$. In conclusion, our systemic analysis suggests that the expression of glypican-3 is highly associated with the diagnosis of pediatric hepatoblastoma.

\section{Acknowledgements}

This study is supported by National Natural Science Foundation (81403434) and Wuhan Youth Science and Technology Morning Program (201150431082).

\section{References}

Abdelgawad IA, Mossallam GI, Radwan NH, et al (2013). Can Glypican3 be diagnostic for early hepatocellular carcinoma among Egyptian patients? Asian Pac J Cancer Prev, 14, 7345-9.

Chan ES,Pawel BR,CoraoDA, et al (2013).Immunohistochemical expression of glypican-3 in pediatric tumors, an analysis of 414 cases. Pediatr Dev Pathol, 16, 272-7.

Chu PG, Ishizawa S, Wu E, et al (2002). Hepatocyte antigen as a marker of hepatocellular carcinoma, an immunohistochemical comparison to carcinoembryonic antigen, CD10, and alpha-fetoprotein. Am J Surg Pathol, 26, $978-88$.

Filmus J, Selleck SB (2001). Glypicans, proteoglycans with a surprise. J Clin Invest, 108, 497-501.

Grisaru S, Cano-Gauci DF, Tee J, et al (2001). Glypican-3 modulates BMP- and FGF-mediated effects during renal branching morphogenesis. Dev Biol, 231, 31-46.

Kim YH, Kang JS (2013). Expression of glypican-3 in mouse embryo stem cells and its derived hepatic lineage cells treated with diethylnitrosamine in vitro. Asian Pac J Cancer Prev, 14, 6341-5.

Kong CS, Appenzeller M, Ferrell LD (2000). Utility of CD34 reactivity in evaluating focal nodular hepatocellular lesions sampled by fine needle aspiration biopsy. Acta Cytol, 44, 218-22.

Hsu HC, Cheng W, Lai PL (1997). Cloning and expression of a developmentally regulated transcript MXR7 in hepatocellular carcinoma, biological significance and temporospatial distribution. Cancer Res, 57, 5179-84.

Ismail H, Broniszczak D, Kaliciński P, et al (2012). Changing treatment and outcome of children with hepatoblastoma, analysis of a single center experience over the last 20 years. J Pediatr Surg, 47, 1331-9.

Lage H, Dietel M, Froschle G, et al (1998). Expression of the novel mitoxantrone resistance associated gene MXR7 in colorectal malignancies. Int J Clin Pharmacol Ther, 36, 58-60.

Lin H, Huber R, Schlessinger D, et al (1999). Frequent silencing of the GPC3 gene in ovarian cancer cell lines. Cancer Res, 59, $807-10$.

Midorikawa Y, Ishikawa S, Iwanari H, et al (2003). Glypican-3, overexpressed in hepatocellular carcinoma, modulates FGF2 and BMP-7 signaling. Int $J$ Cancer, 103, 455-65.

Murthy SS, Shen T, De Rienzo A, et al (2000). Expression of GPC3, an X-linked recessive overgrowth gene, is silenced in malignant mesothelioma. Oncogene, 19, 410-6.

Paine-Saunders S, Viviano BL, Zupicich J, et al (2000). Glypican-3 controls cellular responses to Bmp4 in limb patterning and skeletal development. Dev Biol, 225, 179-87.

Park YN, Yang CP, Fernandez GJ, et al (1998). Neoangiogenesis and sinusoidal 'capillarization' in dysplastic nodules of the liver. Am J Surg Pathol, 22, 656-62.

Piscione TD, Phan T, Rosenblum ND (2001). BMP7 controls collecting tubule cell proliferation and apoptosis via Smad1dependent and -independent pathways. Am J Physiol Renal Physiol, 280, F19-33.

Saikali Z, Sinnett D (2000). Expression of glypican3 (GPC3) in embryonal tumors. Int J Cancer, 89, 418-22.

Watanabe A, Hippo Y, Taniguchi H, et al (2003). An opposing view on WWOX protein function as a tumor suppressor. Cancer Res, 63, 8629-33.

Wang FH, Wen JM, Vong HT, et al (2013). Glypican 3 expression in hepatoblastoma and its diagnostic implication. Zhonghua Bing Li Xue Za Zhi, 42, 806-9.

Xiang YY, Ladeda V, Filmus J (2001). Glypican -3 expression is silenced in human breast cancer. Oncogene, 20, 7408-12.

Yamauchi N, Watanabe A, Hishinuma M, et al (2005). The glypican 3 oncofetal protein is a promising diagnostic marker for hepatocellular carcinoma. Mod Pathol, 18, 1591-8.

Zhang Z, Coomans C, David G (2001). Membrane heparan sulfate proteoglycan-supported FGF2-FGFR1 signaling. Evidence in support of the 'cooperative end structures' model. J Biol Chem, 276, 41921-9.

Zhu ZW, Friess H, Wang L, et al (2001). Enhanced glypican-3 expression differentiates the majority of hepatocellular carcinomas from benign hepatic disorders. Gut, 48, 558-64.

Zynger DL, Gupta A, Luan C, et al (2008). Expression of glypican 3 in hepatoblastoma, an immunohistochemical study of 65 cases. Hum Pathol, 39, 224-30. 